\title{
The K-Property of "Orthogonal” Cylindric Billiards
}

\author{
Domokos Szász ${ }^{1}$ \\ Mathematical Institute of the Hungarian Academy of Sciences, P.O.B. 127, H-1364 Budapest, \\ Hungary
}

Received: 8 March 1993/in revised form: 11 June 1993

\begin{abstract}
Toric billiards with cylindric scatterers (briefly cylindric billiards) generalize the class of Hamiltonian systems of elastic hard balls. In this paper a class of cylindric billiards is considered where the cylinders are "orthogonal" or more exactly: the constituent space of any cylindric scatterer is spanned by some of the (of course, orthogonal) coordinate vectors adapted to the euclidean torus. It is shown that the natural necessary condition for the K-property of such billiards is also sufficient.
\end{abstract}

\section{Introduction. Cylindric Billiards}

It is well-known that Hamiltonian systems of elastic hard balls are isomorphic to certain billiards with cylindric scatterers (cf. [SCh-87], [KSSz-91]). Cylindric billiards, i.e. toric billiards with cylinders as scatterers, belong to the class of semi-dispersing billiards (cf. [SCh-87]). They deserve special attention for they are relatively simple. Indeed, the first semi-dispersing but not dispersing billiard whose ergodicity was shown was a cylindric billiard introduced in [KSSz-89] and the methods developed there for establishing global ergodicity were quite instructive for more complicated models as well.

Consider compact affine subspaces $L^{i}: 1 \leq i \leq N, N \geq 1$ in the $d$-torus $\mathbf{T}^{d}$ (with $\operatorname{dim} L^{i} \leq d-2$ ), and denote $C^{i}:=\left\{Q:=\left(q_{1}, \ldots, q_{d}\right): \operatorname{dist}\left(Q, L^{\imath}\right) \leq r^{\imath}\right\}$, $1 \leq i \leq N$, where each $r^{2}>0$. Denote $\mathbf{Q}:=\mathbf{T}^{d} \backslash\left(\bigcup_{i=1}^{N} C^{i}\right)$ and $M:=\mathbf{Q} \times \mathbf{S}_{d-1}$, where $\mathbf{S}_{d}$ is the $d$-sphere. $M=\{x=(Q, V)\}$ is the phase-space of the billiard given in the domain $\mathbf{Q}$ with cylindric scatterers. The dynamical system $\left(M, S^{\mathbb{R}}, d \mu\right)$, where $S^{\mathbb{R}}$ is the dynamics defined by uniform motion inside the domain and specular reflections at its boundary (the scatterers!) and $d \mu$ is the Liouville measure, is called a cylindric billiard. (As to notions and notations in connection with semi-dispersing billiards we follow the work [KSSz-90].)

\footnotetext{
1 Research supported by the Hungarian National Foundation for Scientific Research, grant No. 1902
} 
In 1991 John Mather [M-91] asked whether a condition that, for instance, the intersection of the constituent subspaces of these cylinders is trivial is sufficient to ensure the ergodicity of the corresponding billiard (in connected components of its phase space, of course). I expect that the answer is yes if, in addition, we exclude the existence of trivial integrals of motion (a natural further assumption is, of course, that the configuration space $\mathbf{Q}$ be connected since otherwise each connected component should belong to different ergonents). To illustrate the appearance of trivial integrals of motion, though the intersection of the constituent subspaces is the zero-space, consider the billiard on the 4-torus with two cylindric scatterers: $C^{1}:=\left\{Q \in \mathbf{T}^{4}:\left(q_{1}^{2}+q_{2}^{2}\right)^{1 / 2} \leq r\right\}$ and $C^{2}:=\left\{\mathbf{Q} \in \mathbf{T}^{4}:\left(q_{3}^{2}+q_{4}^{2}\right)^{1 / 2} \leq r\right\}$. Here, beside the total energy, $v_{1}^{2}+v_{2}^{2}$ and $v_{3}^{2}+v_{4}^{2}$ are also conserved and the billiard is, of course, not ergodic.

The conjecture in such a generality is very strong since it contains the statement of the Boltzmann-Sinai ergodic hypothesis: the system of $N \geq 2$ billiard balls on $\mathbf{T}^{\nu}$, $\nu \geq 2$ is ergodic on connected components of the submanifold of the phase space specified by the trivial integrals of motion (energy, trajectory of the center of mass). This hypothesis is still not settled in general; by recent beautiful results of Simányi [S-92] it is only established for the case $\nu \geq N$ (beside the cases settled earlier: (i) $N=2, \nu \geq 2$, in [SCh-87] and also [S-70], [BS-73]; (ii) $N=3, \nu \geq 2$, in [KSSz-91]; (iii) $N=4, \nu \geq 3$, in [KSSz-92]; we also note that the K-property of a special Hamiltonian system of hard balls was obtained in [BLPS-92]).

Nevertheless we think that the class of cylindric billiards is interesting not only because it contains hard ball systems. Semi-dispersing billiards in general, is too wide and at the same time too wild a category whereas for cylindric billiards it is a realistic aim to obtain transparent necessary and sufficient conditions. In this paper we consider a special class of cylindric billiards: the constituent space of any cylindric scatterer is spanned by some of the coordinate vectors adapted to the orthogonal coordinate system where $\mathbf{T}^{d}$ is given. We call these billiards orthogonal cylindric billiards. In technical terms these billiards are given by a family $C^{j}: 1 \leq j \leq J$ of cylinders $C^{j}:=\sigma_{u^{j}}\left\{\left(q_{1} \ldots, q_{d}\right):\left(\sum_{\imath \in K^{j}} q_{i}^{2}\right)^{1 / 2} \leq r^{j}\right\}$ on the $d$-torus where $\sigma_{u}$ denotes the shift with a vector $u \in \mathbf{T}^{d}$. To concentrate to the essential part of our ideas we assume that the cylinders are disjoint and later, at the end of Sect. 2, we will indicate how this condition can be weakened.

Our main result (announced in [Sz-93]) is the following

Theorem. Assume that, for an orthogonal cylindric billiard with disjoint scatterers, $\mathbf{Q}$ is a connected domain. Then the system is a K-system if and only if $\left\{K^{j}: 1 \leq j \leq J\right\}$ is a connected family of subsets of $\{1, \ldots, d\}$.

The proof is based on the strategy formulated in [KSSz-89] and [KSSz-92]. Indeed, in the spirit of the latter work, the proof of global ergodicity of a semi-dispersing billiard should be based on a suitable definition of richness and then essentially consist of three parts:

(1) geometric-algebraic part for treating neighbourhoods of rich points,

(2) dynamical-topological part for handling the subset of non-rich points,

(3) and, finally, separate arguments for singular trajectories (also settling the ChernovSinai Ansatz).

Our concept of richness, in its spirit, is not very far from that used in [KSSz-92] and in [S-92]. Nonetheless, our method for proving part 1 is novel and is, in fact, 
quite instructive: it makes really clear why the requirement of richness ensures the necessary codimension two property of non-sufficient points. We hope that its idea can be extended to a wider class of models as well. It is worth noting that in [KSSz-92] and [S-92], where hard ball systems are discussed, this part of the proof requires the unpleasant assumption that the dimension of the torus is not too small. In part 2, the failure of the analogue of the theorem of the Appendix of [KSSz-92] requires a new approach. Nevertheless, in the proof of Lemma 4.7, we strongly rely on the proof given in [KSSz-92], and will only present the details where they are different. Finally, the proof given in [KSSz-92] for part 3, in principle, also settles our case. Therefore we omit the proof, and, at the end of Sect. 4, we just put down some calculations to convince the reader that, in fact, this is the case.

The paper is organized as follows: in Sect. 2 we present the notion of richness, formulate the main lemmas and indicate how they imply the theorem. Section 3 is devoted to the geometric-algebraic part 1 by proving Main Lemma 2.2. Section 4 then settles the dynamical-topological part 2 by proving Main Lemma 2.3 and also contains the necessary remarks as to part 3 . According to the traditions, we also need some facts from topological dimension theory, that are summarized in Appendix 1. Appendix 2 is devoted to an elementary analysis of the trivial non-sufficient point of some natural sub-billiards.

For brevity, our exposition relies heavily on earlier works: first of all on [KSSz-92] and [KSSz-89] but also on [KSSz-90] and [KSSz-91]. To help the reader, however, we everywhere provide precise references to the occurrence of the necessary definitions, statements and arguments.

\section{Notion of Richness, Main Lemmas and the Proof}

Likewise as for hard ball systems, the proof is inductive but here induction is only used for brevity of exposition. For $d=2$, a billiard satisfying the conditions of the theorem is necessarily dispersing and thus a $\mathrm{K}$-system. Assume now that any billiard in dimension $d^{\prime}<d$ fulfilling the requirements of the theorem possesses the $\mathrm{K}$-property. Under this condition we will show that the same holds true in dimension $d$ as well. (Though we will have several inductive arguments, the aforeformulated inductive assumption will, in fact, be only used in the proof of Main Lemma 2.3.)

Similarly as in [KSSz-91], $M^{*}$ will denote the set of phase points whose orbits contain an infinite number of collisions among which not more than one is singular. $M^{0} \subset M^{*}$ will be the subset of regular phase points, and $M^{1}:=M^{*} \backslash M^{0}$. Moreover, $\mathscr{S} \mathscr{B}^{+}$will denote the collection of all phase points $x \in \partial M$ for which the reflection, occurring at $x$, is singular (tangential or multiple) and, in the case of a multiple collision, $x$ is supplied with the outgoing velocity $V^{+}$. We remind the reader that a trajectory segment $S^{[a, b]} x$ is called regular (or non-singular) if it does not hit singularities $\left(S^{[a, b]} x \cap \mathscr{S P B}^{+}=\emptyset\right.$; cf. [KSSz-92]).

Consider the regular trajectory segment $S^{[a, b]} x,-\infty \leq a \leq b \leq \infty, x \in M$. Its symbolic collision sequence is the list of subsequent cylinders of collisions $\left(C^{j_{1}}, \ldots, C^{j_{k}}\right), 1 \leq k$ of the trajectory and can be described by the sequence $\left(j_{1}, \ldots, j_{k}\right), 1 \leq j_{l} \leq J, 1 \leq l \leq k$. (If the trajectory hits one or more singularities, then, of course, there are a finite number of such sequences for any finite orbit.)

In the previous section we characterized a cylinder $C$ by a triple $(K, r, u): K \subset$ $\{1, \ldots, d\}, r \in \mathbb{R}^{+}, u \in \mathbf{T}^{d}$. 
Definition 2.1. We say that the trajectory segment $S^{[a, b]} x$ is connected if $\left(K^{j_{1}}, \ldots\right.$, $\left.K^{j_{k}}\right)$ is a connected cover of the set $\{1, \ldots, d\}$. We say that the trajectory segment $S^{[a, b]} x$ is rich if there exists a time $t \in[a, b]$ (with $S^{t} x \in \partial M$ also allowed) such that both trajectory segments $S^{[a, t]} x$ and $S^{[t, b]} x$ are connected. If the trajectory segment hits singularities, then the above properties are required for any trajectory branch.

Finally, the trajectory segment is poor if it is not rich.

Main Lemma 2.2. Assume that the trajectory segment $S^{[a, b]} x$ is regular, $S^{a} x$, $S^{b} x \notin \partial M$ and its trajectory segment is rich. Then there exist a neighbourhood $U \subset M$ of $x$, and a submanifold $N$ such that

(1) $\operatorname{codim} N \geq 2$;

(2) for every $y \in U \backslash N, S^{[a, b]} y$ is sufficient.

(As to the definition of sufficiency cf. Definition 2.4 of [KSSz-92].)

The demonstration of Main Lemma 2.2 will be the content of Sect. 3.

Denote by $M_{p}^{0}$ the subset of poor phase points from $M^{0}$. It would be nice to claim that $M_{p}^{0}$ is residual but there may exist some trivial one-codimensional submanifolds of non-sufficient points for our billiard (and for some auxilliary sub-billiards used in the proof as well). Therefore we should exclude a finite union of one-codimensional submanifolds to obtain $M^{\#}$. Since the introduction of these submanifolds requires some preparation, it is postponed to Appendix 2. Until then it may be instructive to remark that this finite union necessarily contains all phase points whose trajectories never collide in at least one non-trivial sub-billiard of our system. As such these submanifolds are defined by linear conditions on the velocities. Thus we can only claim

Main Lemma 2.3. $M_{p}^{0} \cap M^{\#}$ is a residual subset,

whose demonstration can be found in Sect. 4.

The reader familiar with the technique of establishing global ergodicity of semidispersing billiards already knows that the treatment corresponding to the previous main lemmas for singular points, on one hand, and the verification of the Chernov-Sinai Ansatz, on the other hand, follow from

Main Lemma 2.4. For every cell $C$ of maximal dimension $2 d-3$ in $\mathscr{Y P B}{ }^{+}$, the set $C_{\mathrm{ed}} \subset C$ of all eventually disconnected phase points can be covered by a countable family of closed zero-subsets (with respect to the surface measure $\mu_{C}$ in $C$ ) of $C$.

We say that a point $x \in \mathscr{S P B}^{+}$is eventually disconnected if

(1) the semi-trajectory $S^{\mathbb{R}+} x$ is regular;

(2) there is a number $t_{0}>0$ such that the trajectory segment $S^{\left(t_{0}, \infty\right)} x$ is not connected.

As stated earlier, Main Lemma 2.4 can be derived in almost exactly the same way as Main Theorem 6.1 in [KSSz-92], and, for brevity, we are satisfied by giving some indications at the end of Sect. 4.

Given Main Lemmas 2.2-2.4, the proof of our Theorem is straightforward. It is, first of all, easy to see that the Transversal Fundamental Theorem (for simplicity consider its form given in Theorem 3.4 of [KSSz-92]) is applicable. Indeed, by Main Lemma 2.4, the Chernov-Sinai Ansatz 3.1 holds whereas the fulfillment of the geometric conditions 3.2-3.3 can be seen by standard arguments. Further, our Main Lemmas 2.2-2.4 are clearly the analogues of Main Theorems 4.3, 5.1 and 6.1 of [KSSz-92]. Consequently, they provide the proof of the fact that every connected 
component $\Omega_{1}, \ldots, \Omega_{I}$ of the set $M^{\#}$ introduced in Appendix 2 belongs to one ergodic component. Indeed, this results in the same way as, in section 7 of [KSSz-92], the aforementioned Main Theorems lead to the proof of the Main Theorem of that work. These components, however, can be connected by bundles of orbits of positive measure as is also explained in Appendix 2. This remark already gives the statement of our theorem.

At this point it is time to mention how the assumption on the disjointness of the cylinders can be weakened. If we drop it, then, in principle, there may be trajectories with an infinite number of collisions in a finite time interval. Therefore, as usual, we put, first of all, the following condition (Condition 2.1 from [KSSz-90]): The set of phase points in whose trajectories the moments of reflections accumulate in a finite time interval is a residual subset.

Under this condition the proof of our theorem automatically provides that, in general, the number of ergodic components is finite. Furthermore, the method described in Appendix 2 for connecting different ergodic components definitely works if we, in addition, require e.g. that

$$
\left\{K^{j}: C^{j} \text { does not intersect any other cylinder }\right\}
$$

is itself a connected cover of $\{1, \ldots, d\}$ or for any $1 \leq i<j \leq d\left|K^{i} \cap K^{\jmath}\right| \neq 1$.

\section{Geometric-Algebraic Considerations: Proof of Main Lemma 2.2}

Throughout the whole section we will only consider regular trajectory segments. Main Lemma 2.2 will follow from the following

Lemma 3.1. Assume that the trajectory segment $S^{[a, b]} x$ is a regular, connected one with $S^{a} x, S^{b} x \notin \partial M$. Then there exists a neighbourhood $U \subset M$ of $x$, and $a$ submanifold $N$ such that

(1) $\operatorname{codim} N \geq 1$,

(2) for every $y \in U \backslash N, S^{[a, b]} y$ is sufficient.

Proof of Lemma 3.1. We start by characterizing the neutral subspaces of trajectory segments with simple collision sequences. Assume first that $S^{[a, b]} x, S^{a} x, S^{b} x \notin \partial M$ contains a single collision at time $\tau \in(a, b)$ with the cylinder $C:=\sigma_{u}(B \times A)$, where $B:=\left\{\left(q_{i}: i \in K\right):\left(\sum_{i \in K} q_{i}^{2}\right)^{1 / 2} \leq r\right\}$ and $A:=\left\{\left(q_{i}: i \in K^{c}\right)\right\}$. Now from the definition of the neutral subspace $W_{t}\left(S^{[a, b]} x\right)$ (cf. Def. 2.2 in [KSSz-92]) the following statement is obvious:

Sublemma 3.2. If the trajectory segment $S^{[a, b]} x, S^{a} x, S^{b} x \notin \partial M$ contains a single collison as above, then

$$
W_{\tau^{ \pm}}\left(S^{[a, b]} x\right)=\left\{\alpha P_{K}\left(V_{\tau}^{ \pm}\right)+P_{K^{c}}(Z): \alpha \in \mathbb{R}, Z \in \mathbb{R}^{d}\right\},
$$

where, in general, $P_{K}: \mathbb{R}^{d} \rightarrow \mathbb{R}^{d}$ denotes the orthogonal projection to the subspace $\left\{Q=\left(q_{1}, \ldots, q_{d}\right): q_{i}=0\right.$ if $\left.i \in K^{c}\right\}$, and $V^{ \pm}=p\left(S^{\tau \pm} x\right)$.

Remark 3.4. Clearly, for any $W \in W_{\tau^{ \pm}}\left(S^{[a, b]} x\right)$, the value of $\alpha:=\alpha(W)$ is uniquely determined and $\alpha: W_{\tau^{ \pm}}\left(S^{[a, b]} x\right) \rightarrow \mathbb{R}$ is a linear functional. Indeed, the uniqueness of $\alpha$ is evident from (3.3) whenever $P_{K}(V) \neq 0$. But $P_{K}(V)=0$ is impossible since then $V_{\tau}^{ \pm} \| A$ and no collison occurs with the cylinder $C$. 
This functional is related to the notion of the advance of a collision introduced, in general, for any collision of an arbitrary trajectory segment in [KSSz-92] and in [S92]. It is easy to see that, for the particular case of Lemma 3.1, $\alpha(W)=\frac{\left(P_{K}(W), V\right)}{\left\|P_{K}(V)\right\|^{2}}$.

Next assume that, for some $a<t<b$, both trajectory segments $S^{[a, t]} x$ and $S^{[t, b]} x$ with $\left(S^{a} x, S^{t} x, S^{b} x \notin \partial M\right)$ contain exactly one collision in the times $a<\tau<t<\tau^{\prime}<b$ with the cylinders $C:=\sigma_{u}(B \times A)$ and $C^{\prime}:=\sigma_{u^{\prime}}\left(B^{\prime} \times A^{\prime}\right)$, respectively. Now, if $W \in W_{t}\left(S^{[a, b]} x\right)$, then for some $\alpha, \beta \in \mathbb{R} ; Z, Z^{\prime} \in \mathbb{R}^{d}$

$$
W=\alpha P_{K}\left(V_{t}\right)+P_{K^{c}}(Z)=\beta P_{K^{\prime}}\left(V_{t}\right)+P_{\left(K^{\prime}\right)^{c}}\left(Z^{\prime}\right) \text {. }
$$

This equation then leads to

$$
\begin{aligned}
& (\alpha-\beta) P_{K \cap K^{\prime}}\left(V_{t}\right)+P_{K \backslash K^{\prime}}\left(\alpha V_{t}-Z^{\prime}\right)+P_{K^{\prime} \backslash K}\left(-\beta V_{t}+Z\right) \\
& \quad+P_{\left(K \cup K^{\prime}\right)^{c}}\left(Z-Z^{\prime}\right)=0 .
\end{aligned}
$$

From this relation one readily obtains

Sublemma 3.5. If the trajectory segment $S^{[a, b]} x$ contains exactly two collisions as above, and $P_{K \cap K^{\prime}}\left(V_{t}\right) \neq 0$, then $\alpha=\beta$ and

$$
W_{t}=\alpha P_{K \cup K^{\prime}}\left(V_{t}\right)+P_{\left(K \cup K^{\prime}\right)^{c}}(Z),
$$

where $\alpha \in \mathbb{R}, Z \in \mathbb{R}^{d}$.

A similar argument provides the validity of

Sublemma 3.7. Consider a trajectory segment $S^{[a, b]} x$ with a collision sequence $\left(C_{1}, \ldots, C_{k}\right)\left(C_{\imath} \in\left\{C^{j}: 1 \leq j \leq J\right\}: 1 \leq i \leq k\right)$ and collision times $a<\tau_{1}<\ldots<\tau_{k}<b$. Denote the non-trivial connected components of the family $\left\{K_{i}: 1 \leq i \leq k\right\}$ of subsets by $H_{1}, \ldots, H_{m}:\left|H_{i}\right| \geq 2$ and let $H_{0}:=\{1, \ldots, d\} \backslash \bigcup_{1}^{m} H_{i}$. Assume further that, for any $1 \leq i \leq k-1$ and any $1 \leq l \leq d$, we have $v_{l}^{+}\left(\tau_{\imath}\right) \neq 0$. Then

$$
W_{b}\left(S^{[a, b]} x\right)=\left\{\sum_{g=1}^{m} \alpha_{g} P_{H_{g}}\left(V_{b}\right)+P_{H_{0}}(Z): \alpha_{g} \in \mathbb{R}, Z \in \mathbb{R}^{d}\right\} .
$$

(A similar representation is true for $W_{a}\left(S^{[a, b]} x\right)$, too.)

An important particular case of this sublemma is

Corollary 3.9. In the setup of Sublemma 3.7, assume further that $\left\{K_{i}: 1 \leq i \leq k\right\}$ is a connected cover of $\{1, \ldots, d\}$. Then the trajectory segment is sufficient.

Proof of Sublemma 3.7. We proceed by induction on $k$. The case $k=0$ is trivial. Assume that the statement is true for any $k^{\prime} \leq k$ and any interval $[a, b]$. Let $a<\tau_{1}<, \cdots,<\tau_{k}<b<\tau_{k+1} \leq b^{\prime}$. By our inductive assumption, (3.8) holds true. If $W \in W_{b}\left(S^{\left[a, b^{\prime}\right]} x\right)$, then, on one hand, it should be of the form (3.8) and, on the other hand, of the form $\beta P_{B_{k+1}}\left(V_{b}\right)+P_{A_{k+1}}\left(Z^{\prime}\right)$. The argument of Sublemma 3.5 then gives $\alpha_{g}=\beta$ whenever $H_{g} \cap K_{k+1} \neq \emptyset$. We obtain, moreover, $W_{b}\left(S^{\left[a, b^{\prime}\right]} x\right)$ as a sum analogous to (3.8), from where the required form of $W_{b^{\prime}}\left(S^{\left[a, b^{\prime}\right]} x\right)$ comes by reflection with respect to $C_{k+1}$. 
Corollary 3.9 easily provides now the proof of Lemma 3.1. Indeed, we can assume $b=0$. In a small neighbourhood $U$ of $x$ the collision sequence of $S^{[a, 0]} y$ is a constant. For $(x \in) U$ sufficiently small, let $N:=\left\{y: v_{l}^{+}\left(\tau_{i}\right)=0\right.$ for some $1 \leq i \leq k-1$ and some $1 \leq l \leq d\}$. By Sublemma 3.7, of course, every $y \in U \backslash N$ is sufficient. On the other hand, for any $1 \leq i \leq k-1$ and any $1 \leq l \leq d, \operatorname{codim}\left\{y: v_{l}^{+}\left(\tau_{\imath}\right)=0\right\}$ is 1 since, in a small neighbourhood, $S^{\tau_{i}} y$ is a diffeomorphism. Hence Lemma 3.1.

We can next turn to the

Proof of Main Lemma 2.2. Assume, for simplicity, that $a<0<b, x \in \partial M^{0}$, and the trajectory segments $S^{[a, 0]} x$ and $S^{[0, b]} x$ are connected. Denote their collision sequences and collision times by $\left(C_{-k^{-}}, \ldots, C_{-1}\right),\left(C_{1}, \ldots, C_{k^{+}}\right)$and $a<\tau_{-k^{-}}<\ldots<\tau_{-1}=$ $0=\tau_{1}<\ldots \tau_{k^{+}}<b$, respectively. Introduce, moreover, the notations

$$
\begin{aligned}
& N^{-}:=\left\{y: v_{l}^{-}\left(\tau_{i}\right)=0 \text { for some }-k^{-}+1 \leq i \leq-1 \text { and some } l \in K_{\imath}\right\}, \\
& N^{+}:=\left\{y: v_{l}^{+}\left(\tau_{\imath}\right)=0 \text { for some } 1 \leq i \leq k^{+}-1 \text { and some } l \in K_{i}\right\},
\end{aligned}
$$

and

$$
N:=N^{-} \cap N^{+}
$$

It is evident from the proof of Lemma 3.1, that, if $y \in U \backslash N$, then $y$ is sufficient. It remains to show that codim $N \geq 2$. This statement, however, will be a straight consequence of

Lemma 3.10. Suppose that $S^{[0, b]} x^{0}$ is a regular trajectory segment with a collision sequence $\left(C_{1}, \ldots, C_{k}\right)$. Assume further that either $l \neq 1$, or if $l=1$, then $1 \in \bigcup_{i=1}^{k} K_{i}$. Under these conditions there exists a neighbourhood $U$ of $x^{0}$ such that

$$
\operatorname{codim}_{U}\left\{v_{1}(0)=0, v_{l}(b)=0\right\} \geq 2 \text {. }
$$

Proof of Lemma 3.10. The argument is again inductive. We assume that the claim holds true in any dimension $2 \leq d^{\prime}<d$ for any number $k(\geq 0)$ of collisions, and also in dimension $d$ if the number of collisions $k^{\prime}<k$. Thus we can also suppose that $\left\{K_{\imath}: 1 \leq i \leq k\right\}$ is a cover of $\{1, \ldots, d\}$. If 1 and $l$ belong to different connected components of this cover, then the statement of the lemma is obvious since then the dynamics is the product of two or more dynamics and the subset in (3.10) is a product set of two, one-codimensional submanifolds in the phase spaces of these noninteracting dynamical systems. Consequently, we can and do assume that $\left\{K_{\imath}: 1 \leq i \leq k\right\}$ is a connected cover of $\{1, \ldots, d\}$.

The non-trivial case is, of course, $v_{1}^{0}(0)=0$. Therefore we will consider a product type neighborhood $\bar{U}=\bar{U}_{Q^{0}} \times \bar{U}_{V^{0}}$ in the one-codimensional submanifold determined by the condition $v_{1}(0)=0$ of a point $x^{0}:=\left(Q^{0}, V^{0}\right)$ satisfying $v_{1}^{0}(0)=0$. (Here $\bar{U}_{Q^{0}}$ and $\bar{U}_{V^{0}}$ belong to the configuration and the velocity spaces, respectively.) Denote

$$
\bar{N}:=\left\{y: v_{j}^{+}\left(\tau_{\imath}\right)=0 \text { for some } 1 \leq i \leq k-1 \text { and some } j \in K_{i}\right\} .
$$

By our inductive assumption and Lemma A.1.1

$$
\operatorname{codim}_{\bar{U}} \bar{N}=1 .
$$


Consequently, by Lemma A.1.2, there exists a $\vec{Q} \in \bar{U}_{Q^{0}}$ such that

$$
\operatorname{codim}_{\bar{U}_{V^{0}}}(\bar{N})_{\bar{Q}}=1 \text {. }
$$

Now, by Corollary 3.9, if $V \in \bar{U}_{V^{0}} \backslash(\bar{N})_{\bar{Q}}$, then $S^{[0, b]}(\bar{Q}, V)$ is a sufficient trajectory segment. By sufficiency then, for these $V$, the map of the neighborhood of the origin of $\mathbb{R}^{d-1}$, consisting of vectors $\{d Q ;(d Q, V)=0\}$, into $S_{d-1}$ defined by

$$
p\left(S^{b}(\bar{Q}+d Q, V)\right)
$$

is a local diffeomorphism (following the traditions, $p: M \rightarrow S_{d-1}$ is defined by $p(Q, V):=V)$. Thus the inverse image of the one-codimensional submanifold of $S_{d-1}$ defined by the equation $v_{l}(b)=0$ is also one-codimensional for every $V \in \bar{U} \backslash(\bar{N})_{\bar{Q}}$. Finally, by Lemma A.1.3, we obtain that

$$
\operatorname{codim}_{\bar{U}}\left\{y: p_{l}\left(S^{b} y\right)=0\right\}=1,
$$

and thus, by Lemma A.1.4, we obtain the desired statement.

\section{Dynamical-Topological Part: Proof of Main Lemma 2.3}

We need first a simple characterization of poor trajectory segments.

Lemma 4.1. If a regular orbit $S^{\mathbb{R}} x$ is poor, then there exists a non-collision moment $\tau$ such that neither of the trajectory segments $S^{(-\infty, \tau)} x$ and $S^{(\tau, \infty)} x$ is connected.

Proof. Denote

$$
\begin{aligned}
& T^{-}:=\sup \left\{t: S^{(-\infty, t)} x \text { is not connected }\right\}, \\
& T^{+}:=\inf \left\{t: S^{(t, \infty)} x \text { is not connected }\right\} .
\end{aligned}
$$

The statement is trivial if any of $T^{-}$or $T^{+}$is not finite. If $T^{-}$and $T^{+}$are finite, then they are necessarily collision times. It is easy to see that the orbit $S^{\mathbb{R}} x$ is poor if and only if $T^{-}>T^{+}$, thus providing the statement of the lemma.

For a poor regular orbit, the value of $\tau$ figuring in the statement of Lemma 4.1 can be chosen to be rational. By Property 2.9 of [KSSz-91], it is sufficient to show the validity of

\section{Lemma 4.2.}

$$
M_{n c}:=\left\{x \in M^{0} \backslash \partial M: S^{(-\infty, 0)} x \text { and } S^{(0, \infty)} x \text { are not connected }\right\}
$$

is a residual subset.

Proof of Lemma 4.2. Let $x \in M$. We will repeatedly use Lemma 2.14 of [KSSz91] ensuring that residuality be checked locally. Denote the partition into connected components of the collision graphs of $S^{(-\infty, 0)} x$ and of $S^{(0, \infty)} x$ by

$$
P^{-}:=\left\{H_{1}^{-}, \ldots, H_{i^{-}}^{-}\right\} \text {and by } P^{+}:=\left\{H_{1}^{+}, \ldots, H_{i^{+}}^{+}\right\}
$$

respectively. We wish to separate the cases

(i) $P^{-} \wedge P^{+}=\varepsilon$, where $\varepsilon$ denotes the trivial partition of $\{1, \ldots, d\}$, and (ii) $P^{-} \wedge P^{+} \neq \varepsilon$.

Let us start with the simpler case (ii). Here we can suppose that there exist nonempty $H_{1}$ and $H_{2}$ with $H_{1} \cap H_{2}=\emptyset, H_{1} \cup H_{2}=\{1, \ldots, d\}$ such that they are 
both $P^{-}$- and $P^{+}$-measurable. Under this condition, a variation of the ball-avoiding argument leads us to our goal. Indeed, under the assumptions of the theorem, there exists a $j_{0}$ such that $K^{j_{0}}$ intersects both $H_{1}$ and $H_{2}$. Suppose, for simplicity, that $\left\{K^{j}: K^{j} \subset H_{i}\right\}$ is a connected cover of $H_{i}$ for $i=1,2$. Since we are considering phase points where the dynamics

$$
S^{t} y=\left(S_{H_{1}}^{t} y_{H_{1}}, S_{H_{2}}^{t} y_{H_{2}}\right)
$$

is the product of two non-interacting evolutions, the quantities

$$
\sum_{i \in H_{l}} v_{i}^{2}=E_{l}, \quad l=1,2
$$

are conserved. Fix the values of $E_{1}$ and $E_{2}\left(E_{1}+E_{2}=1\right)$ and restrict our considerations to the submanifold $\Phi:=M_{H_{1}, E_{1}, H_{2}, E_{2}}$ of $M$, where (4.5) holds. The phase points of the aforementioned evolutions necessarily avoid the open subset

$$
B:=\left\{x \in \Phi:\left(\sum_{l \in K^{\jmath_{0}}}\left(q_{0}^{l}-u_{l}^{j_{0}}\right)^{2}\right)^{1 / 2}<r^{j_{0}}\right\} .
$$

By the inductive assumption the systems $\left(M_{H_{i}, E_{\imath}}, S_{H_{i}}^{\mathbb{R}}, \mu_{H_{i}}, i=1,2\right.$ are $\mathrm{K}$ (here we assumed $\left\|H_{i}\right\| \geq 2$; if $\left\|H_{i}\right\|=1$, then the adaptation of the ideas of [KSSz-89] to our situation is even simpler). Their product (4.4) is consequently mixing and, moreover, as it is easy to see, it is a hyperbolic system with singularitites. More exactly, $\operatorname{dim} \Phi=2 d-2$ and for the product $\operatorname{dim} \gamma^{s, u}=d-2$, whereas $\operatorname{dim} \gamma^{0}=2$. Now we claim that

Lemma 4.6. Consider the product of two ergodic semi-dispersing billiards, each satisfying the conditions of the fundamental theorem (Theorem 3.4 from [KSSz-92]). Let $B$ be an open set in the phase space of the product system and $H \subset \mathbb{R}$ such that inf $H=-\infty$, sup $H=\infty$ and, moreover

$$
A_{H}(B):=\left\{x \in \Phi: S^{H} x \cap B=\emptyset\right\} .
$$

Then $\operatorname{codim}_{\Phi} A_{H}(B) \geq 2$.

The proof of the lemma is completely analogous to that of Lemma 3 of [KSSz-89]. The only difference is that here we have two neutral directions corresponding to the time advances of both factor-dynamics. The natural way out is to consider sections in both factors.

By choosing $H=\mathbb{R}$ and applying Lemma 4.6 and then Lemma A.1.7 we obtain the statement of Lemma 4.2 in case (ii).

Turn now to case (i). First we formulate an important lemma. Denote

$$
F:=\left\{y: S^{\mathbb{R}} y \text { is partitioned by } P^{\mp}\right\} .
$$

Lemma 4.7. Suppose that $x \in M^{\#}$ and the intervals $I^{-}:=[a, 0]$ and $I^{+}:=[0, b]$, $(a<0<b)$ are chosen in such a way that $S^{[a, b]} x$ is regular and $S_{H_{i}^{-}}^{I^{-}} x$ and $S_{H_{l}}^{I^{+}} x$ are sufficient for every $1 \leq i \leq i^{-}$, and $1 \leq l \leq i^{+}$, where $\left\{H_{1}^{-}, \ldots, H_{i^{-}}^{-}\right\}:=P\left(S^{[a, 0]} x\right)$ and $\left\{H_{1}^{+}, \ldots, H_{i^{+}}^{+}\right\}=P\left(S^{[0, b]} x\right)$. Then there exists a neighbourhood $U(x \in U)$ such that $F \cap U$ is residual.

Proof of Lemma 4.7. For simplicity of notations, we restrict our attention to the case $i^{-}=i^{+}=2$. The proof is based on the arguments of [KSSz-92] and, for brevity of 
exposition, here we only present the basic calculations to convince the reader that the situation is analogous. Denote $d_{i}^{ \pm}:=\left|H_{i}^{ \pm}\right|, i=1,2$. The main tools of the arguments, as usual, are the stable and unstable local manifolds $\gamma_{e}^{s, u}(y)$ and, moreover, the neutral manifolds $\gamma_{0}(y)$ possessing the maximal possible dimension in almost every point of a sufficiently small neighborhood $U$ of $x$. Denote

$$
\begin{aligned}
\gamma_{H_{\imath}^{+}}^{s}(y):= & C C_{y}\left\{z \in U: q_{j}(z)=q_{\jmath}(y) \text { and } v_{j}(z)=v_{\jmath}(y) \text { for } j \notin H_{\imath}^{+}\right. \text {and } \\
& \left.\operatorname{dist}\left(S_{H_{\imath}^{+}}^{t} z, S_{H_{\imath}^{+}}^{t} y\right) \rightarrow 0 \text { exponentially fast as } t \rightarrow \infty\right\}
\end{aligned}
$$

where $C C_{y}$ denotes the operation of taking the arcwise connected component of a set containing the point $y$, and

$$
\begin{aligned}
\gamma_{H_{\imath}^{-}}^{u}(y):= & C C_{y}\left\{z \in U: q_{j}(z)=q_{j}(y) \text { and } v_{\jmath}(z)=v_{j}(y) \text { for } j \notin H_{\imath}^{-}\right. \text {and } \\
& \left.\operatorname{dist}\left(S_{H_{\imath}^{-}}^{t} z, S_{H_{\imath}^{-}}^{t} y\right) \rightarrow 0 \text { exponentially fast as } t \rightarrow-\infty\right\},
\end{aligned}
$$

and, moreover,

$$
\begin{aligned}
\gamma_{e}^{s}(y) & :=\bigcup_{z \in \gamma_{H_{1}^{+}(y)}^{s}} \gamma_{H_{2}^{+}}^{s}(z) \\
\gamma_{e}^{u}(y) & :=\bigcup_{z \in \gamma_{H_{1}^{u}(y)}^{u}} \gamma_{H_{2}^{-}}^{u}(z)
\end{aligned}
$$

If any element of any partition has just one element, then the previous definitions just define the point $y$ itself and this definition fits into our setup. The maximal dimension of $\gamma_{H_{i}^{+}}^{s}(y)$ is, of course, $d_{i}^{+}-1$ and that of $\gamma_{H_{i}^{-}}^{u}(y)$ is $d_{\imath}^{-}-1$. Consequently, the maximal dimensions of both $\gamma_{e}^{s}(y)$ and of $\gamma_{e}^{u}(y)$ are identically $d-2$. The neutral manifolds are well-defined pieces of the affine neutral subspaces not terminating in $U$. In fact,

$$
\begin{aligned}
\gamma_{0}^{s}(y):= & \left\{z \in U: Q(z)-Q(y)=\alpha_{1} P_{H_{1}^{+}}(p(y))+\alpha_{2} P_{H_{2}^{+}}(p(y))\right. \text { and } \\
& p(z)=p(y)\}, \\
\gamma_{0}^{u}(y):= & \left\{z \in U: Q(z)-Q(y)=\beta_{1} P_{H_{1}^{-}}(p(y))+\beta_{2} P_{H_{2}^{-}}(p(y))\right. \text { and } \\
& p(z)=p(y)\},
\end{aligned}
$$

By definition, $\gamma_{0}(y)$ is the affine subspace spanned by $\gamma_{0}^{s}(y)$ and $\gamma_{0}^{u}(y)$. To calculate the dimension of $\gamma_{0}(y)$ we remark that the only solution of the equation

$$
\alpha_{1} P_{H_{1}^{+}}(V)+\alpha_{2} P_{H_{2}^{+}}(V)=\beta_{1} P_{H_{1}^{-}}(V)+\beta_{2} P_{H_{2}^{-}}(V)
$$

is $\alpha_{1}=\alpha_{2}=\beta_{1}=\beta_{2}$ and, consequently, $\operatorname{dim} \gamma_{0}(y)=3$.

Since $2(d-2)+3=2 d-1$, the methods of [KSSz-92] are applicable assuming we can ensure the transversality of the submanifolds $\gamma_{0}, \gamma_{e}^{s}$ and $\gamma_{e}^{u}$. The situation is, in fact, even simpler here since $2 d-1$ is exactly equal to $\operatorname{dim} M$.

Thus, for this case, the proof works if we establish

Sublemma 4.11. Under the assumptions formulated above

$$
\mathscr{T}_{y} M=\mathscr{T}_{y} \gamma_{0}(y)+\mathscr{T}_{y} \gamma_{e}^{s}(y)+\mathscr{T}_{y} \gamma_{e}^{u}(y)
$$


We note that the statement makes sense and does hold independently of the existence and dimensionality of the figuring submanifolds since the tangent planes are, as usual, determined by the continued fractions.

Proof of Sublemma 4.11. The idea is close to the proof of Lemma 5.20 from [KSSz92] and we only sketch the main steps. The transversality of $\gamma_{e}^{s}(y)$ and of $\gamma_{e}^{u}(y)$ easily follows from convexity arguments. Thus it is sufficient to prove the relation $\mathscr{T}_{y} \gamma_{0}(y) \cap\left[\mathscr{T}_{y} \gamma_{e}^{s}(y)+\mathscr{T}_{y} \gamma_{e}^{u}(y)\right]=0$. Let a perturbation $\Pi=\left\{\left(d q_{i}, d v_{i}\right): 1 \leq i \leq d\right\}$ be a common element of $\mathscr{T}_{y} \gamma_{0}(y)$ and of $\mathscr{T}_{y} \gamma_{e}^{s}(y)+\mathscr{T}_{y} \gamma_{e}^{u}(y)$. The perturbation $\Pi$ can be split into the components $\Pi^{-}=\left\{\left(d q_{i}^{-}, d v_{i}^{-}\right): 1 \leq i \leq d\right\} \in \mathscr{T}_{y} \gamma_{e}^{u}(y)$ and $\Pi^{+}=\left\{\left(d q_{i}^{+}, d v_{i}^{+}\right): 1 \leq i \leq d\right\} \in \mathscr{T}_{y} \gamma_{e}^{s}(y)$.

By convexity arguments

$$
\sum_{H_{\jmath}^{-}} d q_{\imath}^{-} d v_{i}^{-} \geq 0, \quad \sum_{H_{j}^{+}} d q_{\imath}^{+} d v_{\imath}^{+} \leq 0: \quad j=1,2,
$$

with a strict inequality in at least one of the first (and second) pair of relations if $\Pi^{-} \neq 0\left(\Pi^{+} \neq 0\right.$, respectively). By conservation of energy of the subsystems $S_{H_{\jmath}^{-}}^{\mathbb{R}_{-}} y$ and $S_{H_{j}^{+}}^{\mathbb{R}_{+}} y$, we also have

$$
\sum_{H_{\jmath}^{-}} v_{\imath} d v_{i}^{-}=\sum_{H_{j}^{+}} v_{i} d v_{i}^{+}=0 ; \quad j=1,2 .
$$

A consequence of $\Pi \in \mathscr{T}_{y} \gamma_{0}(y)$ is

$$
d v_{i}^{-}+d v_{\imath}^{+}=0 ; \quad 1 \leq i \leq d .
$$

Another consequence, as it is easy to see from (4.10), is that, with suitable $\alpha_{1}, \alpha_{2}$, $\alpha_{3} \in \mathbb{R}$, we have $\Pi=(d Q, 0)$ and

$$
d Q=\alpha_{1} P_{H_{1}^{-}}(V)+\alpha_{2} P_{H_{1}^{+}}(V)+\alpha_{3} V .
$$

By (4.14), the second inequality of (4.12) is equivalent to

$$
\sum_{H_{j}^{+}} d q_{2}^{+} d v_{i}^{-} \geq 0 ; j=1,2 .
$$

Consequently, whenever $I I \neq 0,\left(d Q, d V^{-}\right)>0$. On the other hand, (4.13) trivially implies $\left(d Q, d V^{-}\right)=0$, a contradiction. Hence Sublemma 4.11.

To complete the proof of Lemma 4.7, we note that if either $i^{-}$or $i^{+} \neq 2$, then $\gamma^{s}$ and $\gamma^{u}$ can be defined by a natural generalization of (4.8) and their maximal dimensions are

$$
\begin{aligned}
& \operatorname{dim} \gamma^{u}=\sum_{i=1}^{i^{-}}\left(d_{i}^{-}-1\right)=d-i^{-} \\
& \operatorname{dim} \gamma^{s}=\sum_{i=1}^{i^{+}}\left(d_{i}^{+}-1\right)=d-i^{+}
\end{aligned}
$$


Moreover, the generalization of the arguments around (4.10) easily provides that $\operatorname{dim} \gamma^{0}=i^{-}+i^{+}-1$. Lemma 4.11 holds true in this general case, too, and, since $\left(d-i^{-}\right)+\left(d-i^{+}\right)+\left(i^{-}+i^{+}-1\right)=2 d-1$, the methods of [KSSz-92] are again applicable. Hence Lemma 4.7.

Our final task is to argue that Lemma 4.7 implies the validity of Lemma 4.2 in case (i). To that end we recall again Lemma 2.14 of [KSSz-91] saying that residuality of a set $A$ can be checked locally, i.e. in neighborhoods of points $x \in A$. Fix a point $x \in M_{n c}$. There necessarily exist the limit partitions

$$
P^{-\infty}:=\lim _{t \rightarrow-\infty} P\left(S^{(-\infty, t)} x\right)
$$

and

$$
P^{\infty}:=\lim _{t \rightarrow \infty} P\left(S^{(t, \infty)} x\right)
$$

where $P\left(S^{[a, b]} x\right)$ denotes the partition into connected components of the collision graph of $S^{[a, b]} x$. In general, $P^{-} \preceq P^{-\infty}$ (and $P^{+} \preceq P^{\infty}$ ). By definition, there exist finite rational intervals $I^{-}:=\left[t_{1}^{-}, t_{2}^{-}\right] \subset \mathbb{R}_{-}$and $I^{+}:=\left[t_{1}^{+}, t_{2}^{+}\right] \subset \mathbb{R}_{+}$such that $P\left(S^{I^{\mp}} x\right)=P^{\mp \infty}$ and, moreover, the collision graph of each $S_{H_{2}^{-\infty}}^{\left[t_{1}^{-}-t_{2}^{-}, 0\right]}\left(S_{2}^{t_{2}^{-}} x\right)_{H_{i}^{-\infty}}$, $1 \leq i \leq i^{-}$and of $S_{H_{l}^{\infty}}^{\left[0, t_{2}^{+}-t_{1}^{+}\right]}\left(S^{t_{1}^{+}} x\right)_{H_{l}^{\infty}}, 1 \leq l \leq i^{+}$is rich with respect to the corresponding subdynamics. In virtue of Main Lemma 2.2, by discarding a suitable residual subset, we can assume that all aforementioned trajectory segments are sufficient in the corresponding subdynamics. In case (i), if $S^{\left[t_{2}^{-}, t_{1}^{+}\right]} x$ does not contain any collision, then, by Lemma 4.7 , we are done. If it contains, then let us assume that these collisions occur at times $\left(t_{2}^{-}<\right) \tau_{1}<\ldots \tau_{m}\left(<t_{1}^{+}\right)$with the cylinders $C_{1}, \ldots, C_{m}$. For all $1 \leq i \leq m$ consider the velocities $v_{l}\left(\tau_{i}+\right): l \in K_{\imath}$ and, moreover, the velocities $v_{l}\left(t_{2}^{-}+\right): 1 \leq l \leq d$. By virtue of Lemma 2.10, apart from a residual subset, at most one of all these velocities vanishes. Assume that it is $v_{l}\left(\tau_{i}+\right)$ for some $1 \leq i \leq m, l \in K_{i}$, say. Then choose $\tau_{i}<\bar{t}<\tau_{\imath+1}$, and consider the trajectory segment $S^{\left[t_{1}^{-}, t_{2}^{+}\right]} x$. Now Lemma 4.7 can be applied to the trajectory segments $S^{\left[t_{1}^{-}, \bar{t}\right]} x$ and $S^{\left[\bar{t}, t_{2}^{+}\right]} x$ since, by Lemma 3.7, all the sub-billiard trajectories of these trajectory segments are sufficient (the sub-billiard trajectories should, of course, be taken on connected components of the collision graphs of $S^{\left[t_{1}^{-}, \bar{t}\right]} x$ and of $S^{\left[\bar{t}, t_{2}^{+}\right]} x$ ). This completes the discussion of case (i). Hence Lemma 4.2.

Singular Orbits. To end this section we turn to some thoughts about the proof of Main Lemma 2.4. Our subset $C_{\text {ed }}$ of eventually disconnected points is analogous to the subset $C_{\text {ed }}$ of eventually decomposing points used in [KSSz-92]. For any $t_{0} \in \mathbb{R}$ and any partition $P$ of $\{1, \ldots, d\}$ we can also introduce the subset

$$
\begin{aligned}
C_{\text {ed }}\left(t_{0}, P\right):= & \left\{x \in C_{\text {ed }}:\right. \text { the partition into the connected components } \\
& \text { of the collision graph of } \left.S^{\left(t_{0}, \infty\right)} x \text { is finer than } P\right\} .
\end{aligned}
$$

Assume, for simplicity, that $P=\left\{H_{1}, H_{2}\right\}$ has two nontrivial elements. Now the definitions of $U\left(y_{0}\right), F_{+}, F_{+}^{\prime}$ are evidently the same as in [KSSz-92], whereas $\gamma_{0}^{s}($. is given by (4.5), and further $\gamma_{H_{\imath}}^{s}():. i=1,2$ will appear instead of the manifolds $\gamma_{1,2}^{s}($.$) and y_{3,4}^{s}($.$) . The dimension of$

$$
\gamma_{0}^{s p}(y):=\left\{z \in \gamma_{0}^{s}(y): Q(z)-Q(y) \perp V(y)\right\}
$$


is, of course, 1 and the dimension of

$$
\gamma_{e}^{s}(y):=\bigcup_{z \in \gamma_{H_{1}}^{s}(y)} \gamma_{H_{2}}^{s}(z)
$$

is $d-2$. That of the generate

$$
\gamma_{g}^{s}(y):=\bigcup_{z \in \gamma_{0}^{s p}(y)} \gamma_{e}^{s}(z)=\bigcup_{z \in \gamma_{e}^{s}(y)} \gamma_{0}^{s p}(z)
$$

is then $d-1$. It is easy to see that, with these modifications in the definitions, the proof of Main Theorem 6.1 can literally be copied.

\section{Appendix 1. Some Facts from Topological Dimension Theory}

In the proofs some additional facts are needed compared to those discussed in [KSSz92]. Here only these will be treated otherwise the reader is suggested to consult the aforementioned paper or the monography [E-78]. Moreover, for simplicity of exposition, we do not aim at the widest possible generality. In particular, we always assume that our basic space $M$ is a compact smooth manifold and, moreover, it is the product $M=M_{1} \times M_{2}$ of smooth manifolds.

Denote, for any $A \subset M$,

$$
\operatorname{codim}_{M} A:=\operatorname{dim} M-\operatorname{dim} A,
$$

where $\operatorname{dim} A$ is any of the three classical notions of dimension: the covering, the small inductive or the large inductive dimension.

Lemma A.1.1. Assume that

(i) $F$ is a subset of $M$ with $\operatorname{codim}_{M} F \geq 2$;

(ii) $\bar{M}$ is a submanifold in $M$ with $\operatorname{codim}_{M} \bar{M}=1$.

Then $\operatorname{codim}_{M} F \geq 1$.

Lemma A.1.2. Assume $F$ is a closed subset of $M=M_{1} \times M_{2}$ with $\operatorname{codim}_{M} F \geq 1$. Then there exists an $x \in M_{1}$ such that codim $_{M_{2}} F_{x} \geq 1$, where $F_{x}:=\{y:(\bar{x}, y)$ $\in F\}$.

Lemma A.1.3. Assume that $A$ is an arbitrary subset of $M=M_{1} \times M_{2}$, and

(i) there exists an $L \subset M_{2}$ satisfying $\operatorname{codim}_{M_{2}} L \geq 1$;

(ii) for every $y \in M_{2} \backslash L$ we have $\operatorname{codim}_{M_{1}} A_{y} \geq 1$, where $A_{y}:=\{x:(x, y) \in A\}$. Then $\operatorname{codim}_{M} A \geq 1$.

Lemma A.1.4. Assume

(i) $\bar{U}$ is a smooth submanifold in $M$ with $\operatorname{codim}_{M} \bar{U}=1$;

(ii) $F \subset \bar{U}$ is a subset such that $\operatorname{codim}_{\bar{U}} F \geq 1$.

Then $\operatorname{codim}_{M} F \geq 2$.

These lemmas are simple consequences of the following characterizations of oneand two-codimensional subsets.

Property A.1.5. For every subset $A \subset M, \operatorname{codim}_{M} A \geq 1$ if and only if $\operatorname{Int} A=\emptyset$. 
Property A.1.6. For any closed subset $F \subset M$, the following three conditions are equivalent:

(i) $\operatorname{codim}_{M} F \geq 2$;

(ii) $F \neq M$ and, for every open connected set $G \subset M$, the difference set $G \backslash F$ is also connected;

(iii) For every point $x \in M$ and for any neighborhood $V$ of $x$ in $M$ there exists a smaller neighborhood $W \subset V$ of the point $x$ such that, for every pair of points $y, z \in W \backslash F$, there is a continuous curve $\gamma$ in the set $V \backslash F$ connecting the points $y$ and $z$ and, moreover, Int $F=\emptyset$.

Indeed, Lemmas A.1.1 and A.1.4 are trivial, Lemma A.1.2 is a particular case of Problem 1.9.C of [E-78] and finally for Lemma A.1.3 we can argue as follows:

If $\operatorname{codim}_{M} A=0$, then, by Property A.1.5, $A$ necessarily contains a subset $B_{1} \times B_{2}$, where $B_{1} \subset M_{1}, B_{2} \subset M_{2}$ are ball-like subsets. If $\operatorname{codim}_{M_{2}} L \geq 1$, then, by the same property, $\left(M_{2} \backslash L\right) \cap B_{2} \neq \emptyset$. If $y \in\left(M_{2} \backslash L\right) \cap B_{2}$, then $\operatorname{codim}_{M_{1}} A_{y} \geq 1$, resulting in Int $A_{y}=\emptyset$ contradicting the fact that for $y \in B_{2}, B_{1} \subset A_{y}$.

Finally, we formulate an additional useful property of codimension-two sets from [KSSz-89] (Property 4 from Sect. 4):

Lemma A.1.7. Assume $F$ is a closed subset of $M=M_{1} \times M_{2}$, and for every $y \in M_{2}$, $\operatorname{codim}_{M_{1}} F_{y} \geq 2$. Then $\operatorname{codim}_{M} F \geq 2$.

\section{Appendix 2. Trivial Non-Sufficient Points of the Natural Sub-Billiards}

For a nonempty subset $Z \subset\{1, \ldots, J\}$ consider the sub-billiard $\left(M_{Z}, S_{Z}^{R}, d \mu_{Z}\right)$ on the $d$-torus $\mathbf{T}^{d}$ with the cylinders $\left\{C^{j}: j \in Z\right\}$ as scatterers.

Lemma A.2.1. The subset $\tilde{M}_{Z}$ of phase points from $M_{Z}$ whose orbits have no collisions at all is contained in a finite union $\tilde{M}_{Z}:=\bigcup_{l=1}^{l_{Z}} E_{l}$ of submanifolds such that
for every $l: 1 \leq l \leq l_{Z}$,

(1) $\operatorname{codim}_{M_{Z}} E_{l} \geq 1$;

(2) $E_{l}^{\mathrm{cl}}$ is compact.

The lemma is a direct consequence of

Lemma A.2.2. Let $D \subset \mathbf{T}^{d}$ be any set with a nonempty interior. There exists a finite set of (unit) normal vectors in $\mathbf{R}^{d}$ such that if the trajectory $S^{\mathbf{R}} x$ of a phase point $x=(Q, V)$ never enters $D$, then $V$ is orthogonal to at least one of the given normal vectors.

Proof of Lemma A.2.2. (joint with N. Simányi). Introduce the natural coordinates in $\mathbf{T}^{d}$. We can assume that $D$ is a small ball and $Q=0$. The closure $G=$ $\{\mathbf{R} V\}^{\mathrm{cl}}:=\left\{z \in \mathbf{T}^{d}: \text { for some } t \in \mathbf{R} z=t V\right\}^{\mathrm{cl}}$ of the one-parameter subgroup generated by $V$ is a subtorus (connected closed subgroup) of $\mathbf{T}^{d}$. Furthermore it coincides with $\mathbf{T}^{d}$ whenever the coordinates of $V$ are independent over the rationals. Suppose that this is not the case. Consider the dynamical system $\left(\mathbf{T}^{d}, \Gamma^{\mathbf{R}}, d x\right)$, where $\Gamma_{t} x:=x+t V\left(\bmod Z^{d}\right)$. We want to find the invariant - say $L_{2}$ - functions of $\tilde{\Gamma}_{t}: \tilde{\Gamma}_{t} f(x):=f\left(\Gamma_{t} x\right)$ by standard Fourier methods. To that end we expand $f(x)=\sum_{n} a_{n} \exp (2 \pi i(n, x))$. If $f$ is $\tilde{\Gamma}^{\mathbf{R}}$-invariant, then $(n, V) \neq 0$ implies $a_{n}=0$.

Now $L:=\{n:(n, V)=0\} \subset \mathbf{Z}^{d}$ is a nontrivial subgroup and, in fact, by 
Pontryagin's duality, its dual $L^{*}$ is just the factor group $\mathbf{T}^{d} / G$. This duality also asserts that the subtorus $G$ is exactly the set of all points $x \in \mathbf{T}^{d}$ for which all characters $\exp (2 \pi i(n, x))$ with $n \in L$ take on the value 1 . Consider now the natural projection $\pi: \mathbf{R}^{d} \rightarrow \mathbf{T}^{d}$. The above characterization of $G$ yields

$$
\pi^{-1}(G)=\left\{x \in \mathbf{R}^{d}:(n, x) \text { is an integer for every } n \in L\right\} .
$$

Set the orthogonal direct sum $\mathbf{R}^{d}=W \oplus W^{\perp}$, where the subspace $W$ is the closure of all rational multiples of points from $L$. Of course $\operatorname{dim} W^{\perp}=\operatorname{dim} G$. We denote the dimension of $W$ (i.e. the dimension of $\mathbf{T}^{d} / G$ ) by $k$. It is clear that the group $L$ is a lattice (a discrete subgroup of maximum possible rank $k$ ) in the Euclidean space $W$. The characterization (1) of $\pi^{-1}(G)$ says precisely that $\pi^{-1}(G)=L^{\prime} \oplus W^{\perp}$, where the lattice $L^{\prime}$ in $W$ is the so called dual lattice of $L$, that is

$$
L^{\prime}=\{x \in W:(n, x) \text { is an integer for every } n \in L\} .
$$

Let us denote by $B$ one of the connected components of the inverse image $\pi^{-1}(D)$. Thus, by the assumptions, the set $B$ is a ball of radius $r$ which is disjoint from the set $\pi^{-1}(G)=L^{\prime} \oplus W^{\perp}$. Let us focus on the orthogonal projection $B_{1}=P_{1}(B)$ of the ball $B$ into the subspace $W$. By the disjointness just mentioned, we have that in the $k$-dimensional Euclidean space $W$ there is a ball $B_{1}$ of radius $r$ which is disjoint from the lattice $L^{\prime}$ dual to the lattice $L$. We claim now that there exists a finite number $f(k, r)$ (depending only on the dimension of the Euclidean space and the radius of the ball) such that, under the mentioned circumstances, one can find a nonzero vector $n \in L$ for which $\|n\| \leq f(k, r)$. By the appropriate scaling property of the lattices (i.e. if we multiply $L$ by a positive number $\alpha$, the corresponding dual lattice $L^{\prime}$ will be multiplied by the reciprocal of $\alpha$ ) we can easily see that the sharpest upper estimate $f(k, r)$ has the form $a_{k} / r$, where

$$
\begin{aligned}
a_{k}=\sup \{\|n\|: n \text { is the shortest nonzero vector of a lattice } L \\
\text { whose dual lattice does not intersect some ball of radius one }\} .
\end{aligned}
$$

The value $a_{1}$ is, of course, $\frac{1}{2}$. The questioned finiteness of the numbers $a_{k}$ is positively answered by

Lemma A.2.3. For every integer $k>1$,

$$
a_{k} \leq \sqrt{\frac{1}{4}+\frac{4}{3} a_{k-1}^{2}} .
$$

Proof. Suppose the finiteness of the right-hand-side. Let $L$ be a lattice in the $k$ dimensional Euclidean space $W$ in which the shortest nonzero vector $n_{0}$ is longer than

$$
\sqrt{\frac{1}{4}+\frac{4}{3} a_{k-1}^{2}}
$$

and let $B$ a (say open) ball of unit radius in $W$. We want to show that the dual lattice $L^{\prime}$ intersects $B$. Consider the set

$$
\left\{x \in W:\left(n_{0}, x\right) \text { is an integer }\right\} .
$$

This set is obviously a countable collection of equidistantly placed hyperplanes in $W$, the distance between neighboring such hyperplanes being $1 /\left\|n_{0}\right\|$. Let $H$ be the one among these hyperplanes that has the shortest distance from the center of the ball 
$B$, and let $H_{0}$ be the other amongst these hyperplanes that contains the origin. Let, moreover, $L_{0}^{\prime}$ be the intersection of the dual lattice $L^{\prime}$ with $H_{0}$. It is clear that $L_{0}^{\prime}$ is a lattice (of rank $k-1$ ) in $H_{0}$ and the intersection $L^{\prime} \cap H$ is a translated copy of $L_{0}^{\prime}$. We denote the orthogonal projection of the original lattice $L$ on the subspace $H_{0}$ by $L_{0}$. It is straightforward that $L_{0}$ is a lattice in $H_{0}$ and its dual lattice in the Euclidean space $H_{0}$ is just what we denoted by $L_{0}^{\prime}$. Since every point in the projection $L_{0}$ of $L$ is the projection of an appropriate point of $L$ having distance from $H_{0}$ not bigger than $\frac{\left\|n_{0}\right\|}{2}$, by the Pythagorean Theorem we get that the shortest nonzero vector of the lattice $L_{0}$ can not be shorter than $\frac{\sqrt{3}}{2}\left\|n_{0}\right\|$, i.e. its length is greater than

$$
\frac{\sqrt{3}}{2} \sqrt{\frac{1}{4}+\frac{4}{3} a_{k-1}^{2}} .
$$

By the definition of the number $a_{k-1}$, the lattice $L_{0}^{\prime}$ must intersect every open ball of radius

$$
\frac{a_{k-1}}{\frac{\sqrt{3}}{2} \sqrt{\frac{1}{4}+\frac{4}{3} a_{k-1}^{2}}} .
$$

However, by the choice of the hyperplane $H$ and by the Pythagorean Theorem, the intersection of the unit ball $B$ with $H$ has radius not less than

$$
\sqrt{1-\frac{1}{4\left\|n_{0}\right\|^{2}}}
$$

which number is greater than

$$
\sqrt{1-\frac{1}{4\left(\frac{1}{4}+\frac{4}{3} a_{k-1}^{2}\right)}}
$$

Since this last number is exactly given by (4), the fact that $L^{\prime} \cap H$ is a translated copy of $L_{0}^{\prime}$, immediately implies that the lattice $L^{\prime}$ intersects the ball $B$.

Hence the lemma.

Since $M, M_{Z} \subset \mathbf{T}^{d} \times S_{d-1}(Z \subset\{1, \ldots, J\})$, it makes sense to consider the connected components of the set

$$
M^{\#}:=M \backslash \bigcup_{Z \neq \emptyset}\left(\widetilde{M}_{Z}\right)^{\mathrm{cl}} .
$$

By Lemma A.2.1, their number is finite. Denote them by $\Omega_{1}, \ldots, \Omega_{I}(1 \leq I<\infty)$.

The main body of the paper proves that each $\Omega_{1}, \ldots, \Omega_{I}$ belongs to just one ergodic component. Here we add that they can be connected by bundles of orbits of positive measure. This will be clear from the elementary

Lemma A.2.3. Consider a billiard in $\mathbf{Q}:=T^{d} \backslash C$, where $C$ is a spherical scatterer. Vary the configuration component $Q$ of a phase point $\left(Q, V^{-}\right)$by keeping its velocity coordinate fixed. Then, as $Q$ varies, $p\left(\tau^{+}\left(Q, V^{-}\right)\right)$is a mapping with maximal rank $d-1$ (as usual, $p\left(\tau^{+}\left(Q, V^{-}\right)\right)$denotes the outgoing velocity after the first collision of the phase point $\left.\left(Q, V^{-}\right)\right)$. 
Indeed, each $\Omega_{i}: 1 \leq i \leq I$ is defined by a finite number of linear inequalities on the velocities. On the other hand, by Lemma A.2.3, for any $C^{j}: 1 \leq j \leq J$ the following statement is true: for any fixed values of $v_{\imath}: i \notin K^{j}$ and for any pair $\left(v_{i}^{-}: i \in K^{j}\right)$ and $\left(v_{i}^{+}: i \in K^{j}\right)$ such that for some pair $Q^{-}, Q^{+}$

$$
\begin{aligned}
& x^{-}:=\left(Q^{-},\left\{v_{i}: i \notin K^{\jmath} ; v_{i}^{-}: i \in K^{j}\right\}\right) \in M^{\#}, \\
& x^{+}:=\left(Q^{+},\left\{v_{i}: i \notin K^{\jmath} ; v_{i}^{+}: i \in K^{j}\right\}\right) \in M^{\#},
\end{aligned}
$$

where for some $\varepsilon>0 \quad x^{+}=S^{\varepsilon} x^{-}$and $S^{[0, \varepsilon]} x^{-}$contains exactly one collison, it is true that suitable neighbourhoods of the phase points $x^{-}$and $x^{+}$can be connected by bundles of orbits of positive measure.

It is also clear that, if for some $j_{1}$ and $j_{2}, K^{j_{1}} \cap K^{j_{2}} \neq \emptyset$, then the aforeformulated statement also holds for the union $K^{\jmath_{1}} \cup K^{\jmath_{2}}$ instead of $K^{\jmath}$. Since $K^{j}: 1 \leq j \leq J$ is a connected cover, we also obtain by induction that, in fact, every connected component of $M^{\#}$ can be connected to any other by bundles of trajectories of positive measure. Thus $M^{\#}$ makes, indeed, just one ergodic component.

Acknowledgements. The author is indebted to John Mather for raising the question which was actually the starting point of this research. Sincere thanks are due to Nándor Simányi for his most critical reading of the first version of the text and for his remark that the analogue of the theorem of the Appendix of [KSSz-92] fails to hold for cylindric billiards. Useful remarks of the referee are also acknowledged. Finally, the author expresses his gratitude for the hospitality of the IHES, Bures-surYvette where, on one hand, Sect. 3 was written during the author's stay in November 1992 and, on the other hand, the final version was prepared in April 1993.

\section{References}

[BLPS-92] Bunimovich, L.A., Liverani, C., Pellegrinotti, A., Sukhov, Yu.: Special systems of hard balls that are ergodic. Commun. Math. Phys 146, 357-396 (1992)

[BS-73] Bunimovich, L.A., Sinai, Ya. G.: On a fundamental theorem in the theory of dispersing billiards. Mat. Sbornik 90, 415-431 (1973)

[E-78] Engelking, R.: Dimension Theory. North-Holland, 1978

[KSSz-89] Krámli, A., Simányi, N., Szász, D.: Ergodic properties of semi-dispersing billiards. I. Two cylindric scatterers in the 3-D torus. Nonlinearity 2, 311-326 (1989)

[KSSz-90] Krámli, A., Simányi, N., Szász, D.: A "transversal" fundamental theorem for semidispersing billiards. Commun. Math. Phys. 129, 535-560 (1990)

[KSSz-91] Krámli, A., Simányi, N., Szász, D.: The K-property of three billiard balls. Ann. Math.133, 37-72 (1991)

[KSSz-92] Krámli, A., Simányi, N., Szász, D.: The K-Property of four billiard balls. Commun. Math. Phys. 144, 107-148 (1992)

[M-91] Mather, J.: Oral communication

[S-70] Sinai, Ya. G.: Dynamical systems with elastic reflections. Usp. Mat. Nauk 25, 141-192 (1970)

[S-92] Simányi, N.: The K-property of $N$ billiard balls I. Invent. Math. 108, 521-548 (1992); II., ibidem 110, 151-172 (1992)

[SCh-87] Sinai, Ya. G., Chernov, N.I.: Ergodic properties of some systems of 2-D discs and 3-D spheres. Usp. Mat. Nauk 42,153-174 (1987)

[Sz-93] Szász, D.: Ergodicity of classical hard balls. Physica A 194, 86-92 (1993) 
